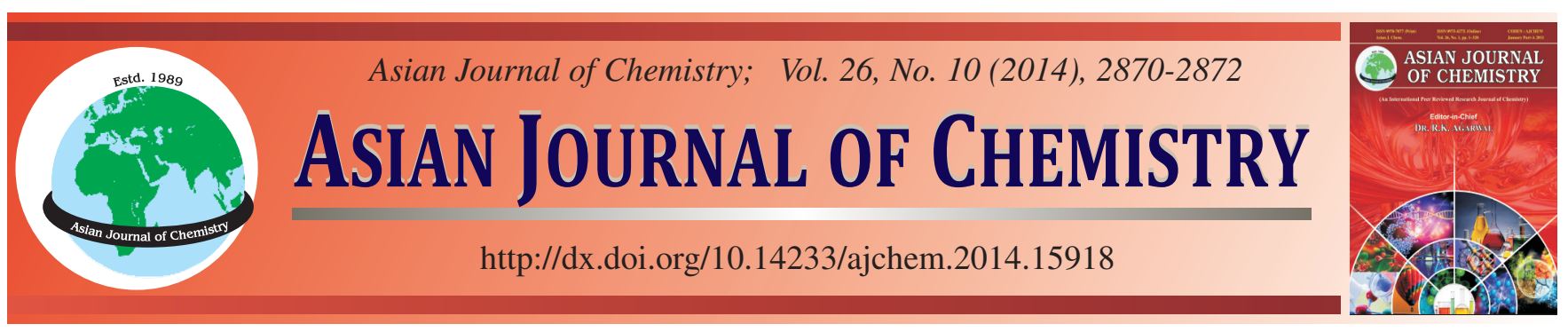

\title{
Pyrazolopyridines II: Synthesis and Antibacterial Screening of 6-Aryl-3-methyl-1-phenyl-1H-pyrazolo[3,4-b]pyridine-4-carboxylic Acids
}

Tahir Maqbool ${ }^{1}$, Areesha Nazeer ${ }^{2}$, Muhammad Naeem Khan ${ }^{3},{ }^{3}$, Mark C. Elliott $^{4}$, Misbahul Ain Khan $^{1,2}$, Muhammad Ashraf ${ }^{5}$, Muhammad Nasrullah ${ }^{1}$, Shafia Arshad ${ }^{5}$ and Munawar Ali Munawar ${ }^{2}$

${ }^{1}$ Department of Chemistry, The Islamia University of Bahawalpur, Bahawalpur, Pakistan
${ }^{2}$ Institute of Chemistry, University of the Punjab, Lahore, Pakistan
${ }^{3}$ Applied Chemistry Research Centre, PCSIR Laboratories Complex, Lahore, Pakistan
${ }^{4}$ School of Chemistry, Cardiff University, Cardiff, UK
${ }^{5}$ Department of Biochemistry and Biotechnology, The Islamia University of Bahawalpur, Bahawalpur, Pakistan

*Corresponding author: Email: changwani_1@yahoo.com

Received: 15 June 2013;

Accepted: 26 December 2013;

Published online: 10 May 2014;

AJC-15137

5-Amino-3-methyl-1-phenyl-1H-pyrazole was prepared to react with various aromatic aldehydes and pyruvic acid to afford 6-aryl-3methyl-1-phenyl-1 $H$-pyrazolo[3,4- $b$ ]pyridine-4-carbxylic acids. The prepared compounds were characterized by Mass, IR, ${ }^{1} \mathrm{H},{ }^{13} \mathrm{C}$ NMR spectra and $\mathrm{CHN}$ analysis data. Various 6-aryl-3-methyl-1-phenyl-1H-pyrazolo[3,4-b]pyridine-4-carbxylic acids synthesized during this work were also evaluated for their antibacterial activities. Some of these compounds were found to be good antibacterial agents.

Keywords: Pyrazolopyridines, Doebner Reaction, Schiff's bases, Pyruvic acid.

\section{INTRODUCTION}

Pyrazolopyridines are well known for their biological activities. These are found to be good antiviral agents, potent P-38 kinase and HIV reverse transcriptases ${ }^{1-4}$. A number of pyrazolopyridines have also been reported as good vasodilating, anti inflammatory, analgesics, antipyretic, antimalarial and antibacterial compounds $s^{5-11}$. We have already presented the synthesis of some novel ethyl 1,6-diaryl-1H-pyrazolo[3,4$b$ ]pyridine-4-carboxylates ${ }^{12}$ which may be considered as ester analogs of cincophen. In continuation of our work, we would now like to report our success with their corresponding 4carboxylic acids and their antibacterial screening.

\section{EXPERIMENTAL}

The chemicals used were commercially available from Merck or Fluka and were used as such. However when needed were purified using normal techniques. The solvents used were distilled and dried. FTIR spectra were recorded on Bruker Tensor-27. Melting points were taken on a GallenKamp melting point apparatus and are uncorrected. The ${ }^{1} \mathrm{H}$ and ${ }^{13} \mathrm{C}$ NMR spectra were taken on Bruker DPX instrument at $400 \mathrm{MHz}$ and $100 \mathrm{MHz}$, respectively.

Preparation of 6-Aryl-1H-pyrazolo[3,4- $b]$ pyridine-4-carboxylic acids (2a-2h) (Table-1)

General procedure: A mixture of equimolar amounts of respective 5 -aminopyrazole $(2.0 \mathrm{mmol})$, appropriate aromatic

\begin{tabular}{|c|c|c|c|}
\hline \multicolumn{4}{|c|}{$\begin{array}{l}\text { TABLE-1 } \\
\text { 6-ARYL-3-METHYL-1-PHENYL-1 } H \text {-PYRAZOLO[3,4- } \\
\text { b]PYRIDINE-4-CARBOXYLIC ACIDS (2a-2h) }\end{array}$} \\
\hline Compound & $X$ & Yield (\%) & m.p. $\left({ }^{\circ} \mathrm{C}\right)$ \\
\hline $2 a$ & $\mathrm{H}$ & 35 & $272-274^{*}$ \\
\hline $2 b$ & $2-\mathrm{Cl}$ & 46 & $214-216$ \\
\hline $2 c$ & $4-\mathrm{Cl}$ & 47 & $294^{* *}$ \\
\hline 2d & $3-\mathrm{CH}_{3}$ & 38 & $176-178$ \\
\hline $2 e$ & $4-\mathrm{OC}_{2} \mathrm{H}_{5}$ & 34 & 192-194 \\
\hline $2 f$ & $3-\mathrm{NO}_{2}$ & 29 & $210-212$ \\
\hline $2 \mathrm{~g}$ & $4-F$ & 46 & $266-268$ \\
\hline $2 \mathrm{~h}$ & $4-\mathrm{OH}$ & 40 & $260-262$ \\
\hline
\end{tabular}

aldehydes ( $2 \mathrm{mmol}$ ) and one drop of $\mathrm{HCl}$ was heated without any solvent at $100{ }^{\circ} \mathrm{C}$ on an oil bath. It was followed by successive addition of an equimolar amount of pyruvic acid and $3 \mathrm{~mL}$ of glacial acetic acid. The mixture was further heated under reflux for $2 \mathrm{~h}$, allowed to cool to room temperature, filtered and dried. The crude compounds were recrystallized from ethanol.

In the case of solid aldehydes (products $\mathbf{2 c}$ and $\mathbf{2 f}$ ), the Schiff bases were prepared by heating the mixture under reflux in ethanol $(5 \mathrm{~mL})$. Ethanol was evaporated and followed by the same general procedure as described above.

1,6-Diphenyl-3-methyl-1H-pyrazolo[3,4-b]pyridine-4carboxylic acid. (2a): Yield: 35 \%; m.p.: 270-274 ${ }^{\circ}$ C. lit. ${ }^{11}$ 
285-287 ${ }^{\circ} \mathrm{C}$; IR (KBr, $\left.v_{\max }, \mathrm{cm}^{-1}\right): 3170(\mathrm{O}-\mathrm{H}) ; 3060-2950$ $(\mathrm{C}-\mathrm{H}) ; 1714(\mathrm{C}=\mathrm{O}) ;{ }^{1} \mathrm{H}$ NMR $\left(\mathrm{CDCl}_{3}\right): \delta 2.81\left(\mathrm{~s}, 3 \mathrm{H}, \mathrm{CH}_{3}\right)$, 7.35-8.36 (m, 11H, ArH), 13.81 (br.s, $1 \mathrm{H}, \mathrm{COOH}),{ }^{13} \mathrm{C}$ NMR $\left(\mathrm{CDCl}_{3}\right): \delta 15.89\left(\mathrm{CH}_{3}\right), 112.00,115.46,121.48,126.57$, $127.43,128.82,128.93,129.64,134.90,138.32,139.08$, 142.92, 152.02, 156.85, $167.41(\mathrm{C}=\mathrm{O})$; MS: $m / z(\%)=$ Calcd. for $\left(\mathrm{C}_{20} \mathrm{H}_{15} \mathrm{~N}_{3} \mathrm{O}_{2}\right)\left[\mathrm{M}^{+}\right]$: 329.12; Found: 329.0 Anal. Calcd. for $\mathrm{C}_{20} \mathrm{H}_{15} \mathrm{~N}_{3} \mathrm{O}_{2}$ : C, 72.94; H, 4.59; N, 12.76. Found: C, 72.54; H, $5.09 ; \mathrm{N}, 13.07$.

6-(3'-Chlorophenyl)-3-methyl-1-phenyl-1H-pyrazolo[3,4-b]pyridine-4-carboxylic acid. (2b): Yield: $45 \%$; m.p.: 214-216 ${ }^{\circ} \mathrm{C}$; IR $\left(\mathrm{KBr}, \mathrm{v}_{\max }, \mathrm{cm}^{-1}\right): 3152(\mathrm{O}-\mathrm{H})$; 3030-2920 (C$\mathrm{H}) ; 1705(\mathrm{C}=\mathrm{O}) ; 1585(\mathrm{C}=\mathrm{C}) ;{ }^{1} \mathrm{H}$ NMR (DMSO): $\delta 2.70(\mathrm{~s}$, $\left.3 \mathrm{H}, \mathrm{CH}_{3}\right), 7.3-8.3(\mathrm{~m}, 10 \mathrm{H}, \mathrm{ArH}) ;{ }^{13} \mathrm{C} \mathrm{NMR}$ (DMSO): $\delta 15.96$ $\left(\mathrm{CH}_{3}\right), 115.31,121.47,125.55,125.93,127.49,128.96,129.66$, 130.06, 134.88, 135.01, 139.01, 140.09, 142.90, 155.15, 167.16 $(\mathrm{C}=\mathrm{O})$; MS: $m / z(\%)=$ Calcd. for $\left(\mathrm{C}_{20} \mathrm{H}_{14} \mathrm{~N}_{3} \mathrm{O}_{2} \mathrm{Cl}\right)\left[\mathrm{M}^{+}\right]$: 363.08 \& [M + 2]: 365.08; Found: $363.0 \&$ 365.1; Anal. Calcd. for $\mathrm{C}_{20} \mathrm{H}_{14} \mathrm{~N}_{3} \mathrm{O}_{2} \mathrm{Cl}$ : C, 66.03; H, 3.88; N, 11.55. Found: C, 65.74; $\mathrm{H}, 3.78 ; \mathrm{N}, 11.34$.

6-(4'-Chlorophenyl)-3-methyl-1-phenyl-1H-pyrazolo[3,4-b]pyridine-4-carboxylic acid. (2c): Yield: $47 \%$; m.p.: $294{ }^{\circ} \mathrm{C}$ lit. ${ }^{11} 280-282{ }^{\circ} \mathrm{C}$; IR (KBr, $\left.v_{\max }, \mathrm{cm}^{-1}\right): 3148(\mathrm{O}-\mathrm{H})$; 3055-2940 (C-H); $1702(\mathrm{C}=\mathrm{O}) ; 1582(\mathrm{C}=\mathrm{C})$; ${ }^{1} \mathrm{H}$ NMR (DMSO); $\delta 2.70\left(\mathrm{~s}, 3 \mathrm{H}, \mathrm{CH}_{3}\right), 7.30-8.4(\mathrm{~m}, 9 \mathrm{H}, \mathrm{ArH}), 8.18(\mathrm{~s}$, $1 \mathrm{H}, \mathrm{H}-5) ;{ }^{13} \mathrm{C}$ NMR (DMSO): $\delta 15.56\left(\mathrm{CH}_{3}\right), 111.71,114.37$, $120.77,125.86,129.05,129.17,134.98,136.19,136.34$, 138.73,142.28, 151.24, 154.78, $166.41(\mathrm{C}=\mathrm{O})$; MS: $\mathrm{m} / z(\%)$ $=$ Calcd. for $\left(\mathrm{C}_{20} \mathrm{H}_{14} \mathrm{~N}_{3} \mathrm{O}_{2} \mathrm{Cl}\right)\left[\mathrm{M}^{+}\right]: 363.08 \&[\mathrm{M}+2]: 365.08$; Found: $363.1 \&$ 365.1; Anal. Calcd. for $\mathrm{C}_{20} \mathrm{H}_{14} \mathrm{~N}_{3} \mathrm{O}_{2} \mathrm{Cl}$ : C, 66.03; H, 3.88; N, 11.55. Found: C, 66.03; H, 3.74; N, 11.60.

3-Methyl-1-phenyl-6-m-tolyl-1H-pyrazolo[3,4- $b]$ pyridine-4-carboxylic acid. (2d) : Yield: $38 \%$; m.p.: 176$178{ }^{\circ} \mathrm{C}$; IR (KBr, $\left.v_{\max }, \mathrm{cm}^{-1}\right)$ : $3120(\mathrm{O}-\mathrm{H})$; 3030-2920 (C-H); $1715(\mathrm{C}=\mathrm{O}) ;{ }^{1} \mathrm{H}$ NMR $\left(\mathrm{CDCl}_{3}\right): \delta 2.46\left(\mathrm{~s}, 3 \mathrm{H}, \mathrm{PhCH}_{3}\right), 2.82$ (s, 3H, $\mathrm{CH}_{3}$ ), 7.26-8.35 (m, 9H, ArH), 8.22 (s, 1H, H-5); ${ }^{13} \mathrm{C}$ NMR $\left(\mathrm{CDCl}_{3}\right): \delta 16.43\left(\mathrm{CH}_{3}\right), 21.63\left(\mathrm{ArCH}_{3}\right), 111.97,116.62$, $124.65,125.87,126.84,128.91,129.01,129.85,130.79$, 132.88, 138.16, 138.60, 139.30, 142.64, 152.21, 156.99, $169.45(\mathrm{C}=\mathrm{O})$; MS: $m / z(\%)=$ Calcd. for $\left(\mathrm{C}_{21} \mathrm{H}_{17} \mathrm{~N}_{3} \mathrm{O}_{2}\right)\left[\mathrm{M}^{+}\right]$: 343.13; Found: 343.2; Anal. Calcd. for $\mathrm{C}_{21} \mathrm{H}_{17} \mathrm{~N}_{3} \mathrm{O}_{2}$ : C, 73.45; H, 4.99; N, 12.24. Found: C, 73.69; H, 5.24; N, 11.78.

6-(4'-Ethoxyphenyl)-3-methyl-1-phenyl-1H-pyrazolo[3,4-b]pyridine-4-carboxylic acid. (2e): Yield: $34 \%$; m.p.:192-194 ${ }^{\circ} \mathrm{C}$; IR (KBr, $\left.v_{\max }, \mathrm{cm}^{-1}\right)$ : $3220(\mathrm{O}-\mathrm{H}) ; 3040-2930$ $(\mathrm{C}-\mathrm{H}) ; 1713(\mathrm{C}=\mathrm{O}) ; 1572(\mathrm{C}=\mathrm{C}) ;{ }^{1} \mathrm{H}$ NMR (DMSO): $\delta 1.45$ $\left(\mathrm{t}, 3 \mathrm{H}, \mathrm{OCH}_{2} \mathrm{CH}_{3}, J=6.8\right), 2.80\left(\mathrm{~s}, 3 \mathrm{H}, \mathrm{CH}_{3}\right), 4.11(\mathrm{q}, 2 \mathrm{H}$, $\left.\mathrm{OCH}_{2} \mathrm{CH}_{3}, J=6.8\right), 7.0-8.35(\mathrm{~m}, 10 \mathrm{H}, \mathrm{ArH}) ;{ }^{13} \mathrm{C} \mathrm{NMR}$ (DMSO): $\delta 14.38\left(\mathrm{OCH}_{2} \mathrm{CH}_{3}\right), 15.58\left(\mathrm{CH}_{3}\right), 63.45\left(\mathrm{OCH}_{2}\right)$, $111.22,114.57,121.56,124.93,125.93,128.93,129.82$, $130.50,134.69,138.95,142.79,151.89,156.52,160.38$, $167.32(\mathrm{C}=\mathrm{O})$; MS: $m / z(\%)=$ Calcd. for $\left(\mathrm{C}_{22} \mathrm{H}_{19} \mathrm{~N}_{3} \mathrm{O}_{3}\right)\left[\mathrm{M}^{+}\right]$: 373.14; Found: 373.1; Anal. Calcd. for $\mathrm{C}_{22} \mathrm{H}_{19} \mathrm{~N}_{3} \mathrm{O}_{3}$ : C, 70.76; H, 5.13; N, 11.25. Found: C, 70.31; H, 5.52; N, 11.16.

3-Methyl-6-(3'-nitrophenyl)-1-phenyl-1H-pyrazolo[3,4-b]pyridine-4-carboxylic acid. (2f): Yield: $29 \%$; m.p.: 210-212 ${ }^{\circ} \mathrm{C}$; IR $\left(\mathrm{KBr}, v_{\max }, \mathrm{cm}^{-1}\right): 3130(\mathrm{O}-\mathrm{H})$; 3050-2930 $(\mathrm{C}-\mathrm{H}) ; 1700(\mathrm{C}=\mathrm{O}) ; 1596(\mathrm{C}=\mathrm{C}), 1525,1345\left(\mathrm{NO}_{2}\right) ;{ }^{1} \mathrm{H}$ NMR (DMSO): $\delta 2.71\left(\mathrm{~s}, 3 \mathrm{H}, \mathrm{CH}_{3}\right), 7.3-9.0(\mathrm{~m}, 10 \mathrm{H}, \mathrm{ArH}) ;{ }^{13} \mathrm{C}$
NMR (DMSO): $\delta 15.52\left(\mathrm{CH}_{3}\right), 112.22,114.70,120.70$, 120.97, 121.76, 124.5, 126.11, 129.04, 129.19, 130.70, 133.59, 138.59, 139.10, 142.38 148.49, 151.11, 153.59, $166.34(\mathrm{C}=\mathrm{O})$; MS: $m / z(\%)=$ Calcd. for $\left(\mathrm{C}_{20} \mathrm{H}_{14} \mathrm{~N}_{4} \mathrm{O}_{4}\right)\left[\mathrm{M}^{+}\right]: 374.10$; Found: 374.0; HRMS (EI): Calcd. for $\left(\mathrm{C}_{20} \mathrm{H}_{14} \mathrm{~N}_{4} \mathrm{O}_{4}\right)\left[\mathrm{M}^{+}\right]$: 374.1671 ; Found: 374.1626.

6-(4'-Fluorophenyl)-3-methyl-1-phenyl-1H-pyrazolo [3,4-b]pyridine-4-carboxylic acid. (2g): Yield: $46 \%$; m.p.: 266-268 ${ }^{\circ} \mathrm{C}$; IR (KBr, $\left.v_{\max }, \mathrm{cm}^{-1}\right): 3160(\mathrm{O}-\mathrm{H})$; 3060-2950 $(\mathrm{C}-\mathrm{H}) ; 1710(\mathrm{C}=\mathrm{O}) ; 1593(\mathrm{C}=\mathrm{C}) ;{ }^{1} \mathrm{H}$ NMR (DMSO): $\delta 2.70$ (s, 3H, $\left.\mathrm{CH}_{3}\right), 7.30-8.35$ (m, 9H, ArH), 8.15 (s, 1H, H-5); ${ }^{13} \mathrm{C}$ NMR (DMSO): $\delta 15.58\left(\mathrm{CH}_{3}\right), 111.48,114.31,115.86$, $116.08,120.75,125.84,129.18,129.60,129.69,134.05$, 136.18, 138.79, 142.27, 151.27, 155.04, 162.19, 164.66, $166.49(\mathrm{C}=\mathrm{O})$; MS: $m / z(\%)=$ Calcd. for $\left(\mathrm{C}_{20} \mathrm{H}_{14} \mathrm{~N}_{3} \mathrm{O}_{2} \mathrm{~F}\right)\left[\mathrm{M}^{+}\right]$: 347.11; Found: 347.1; HRMS (EI): Calcd. for $\left(\mathrm{C}_{20} \mathrm{H}_{14} \mathrm{~N}_{3} \mathrm{O}_{2} \mathrm{~F}\right)$ $\left[\mathrm{M}^{+}\right]$: 347.1072 ; Found: 347.1097.

6-(4'-Hydroxyphenyl)-3-methyl-1 - phenyl-1H pyrazolo[3,4-b]pyridine-4-carboxylic acid. (2h): Yield: 40 \%; m.p.: 260-262 ${ }^{\circ} \mathrm{C}$; IR (KBr, $\left.v_{\max }, \mathrm{cm}^{-1}\right)$ : 3298 (O-H); 30252940 (C-H); $1706(\mathrm{C}=\mathrm{O}) ; 1594(\mathrm{C}=\mathrm{C}) ; 3298(\mathrm{OH}) ;{ }^{1} \mathrm{H}$ NMR (DMSO): $\delta 2.70$ (s, 3H, $\mathrm{CH}_{3}$ ), 6.95-8.35 (m, 9H, ArH), 8.05 (s, 1H, H-5), 9.98 (br. s, $1 \mathrm{H}, \mathrm{OH}) ;{ }^{13} \mathrm{C}$ NMR (DMSO): $\delta 15.58$ $\left(\mathrm{CH}_{3}\right), 110.77,113.66,115.86,120.59,125.56,128.39$, $128.90,129.15,135.93,138.97,142.21,151.47,156.25$, 159.57, $166.68(\mathrm{C}=\mathrm{O})$; MS: $m / z(\%)=$ Calcd. for $\left(\mathrm{C}_{20} \mathrm{H}_{15} \mathrm{~N}_{3} \mathrm{O}_{3}\right)$ $\left[\mathrm{M}^{+}\right]$: 345.11; Found: 345.11; Anal. Calcd. for $\mathrm{C}_{20} \mathrm{H}_{15} \mathrm{~N}_{3} \mathrm{O}_{3}: \mathrm{C}$, 69.56; H, 4.38; N, 12.17. Found: C, 69.29; H, 4.59; N, 12.08.

\section{RESULTS AND DISCUSSION}

6-Aryl-3-methyl-1-phenyl-1H-pyrazolo[3,4- $b$ ]pyridine4-carboxylic acids (2a-2h) were prepared according to Scheme-I, from 5-amino-3-methyl-1-phenyl-1H-pyrazole ${ }^{13} .5$ Aminopyrazoles were prepared to react with various aromatic aldehydes to obtain respective Schiff's bases which were, without isolation, subjected to condensation with pyruvic acid to give 3-methyl-1-phenyl-6-aryl-1H-pyrazolo[3,4-b]pyridine-4carboxylic acids (2a-2h). The yields of the recrystallized products are in the range of 29 to $47 \%$; the crude yields are although much higher. No attempts, at this stage, were made to optimize them. All the products obtained as fine crystals, are stable compounds at room temperature and were characterized by IR, ${ }^{1} \mathrm{H},{ }^{13} \mathrm{C}$ NMR spectra.

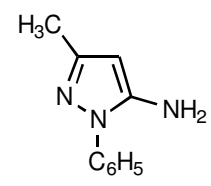

(1)

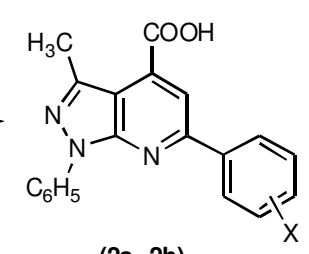

(2a- 2h)
Scheme-I

Spectral characterization of synthesized compounds: In all these compounds $\mathrm{C}=\mathrm{O}$ group of acid functionality at 4th position showed an absorption band in the IR spectral range of $1715-1700 \mathrm{~cm}^{-1}$. In the ${ }^{1} \mathrm{H}$ NMR spectra a singlet in the range of 2.70 to $2.80 \mathrm{ppm}$ confirmed the presence of three proton of methyl group present at position 3 of pyrazole ring. 
TABLE-2

\begin{tabular}{ccccccc}
\hline \multirow{2}{*}{ Compound } & $\begin{array}{c}\text { B. subtilis (+) } \\
\text { MIC }_{50}\end{array}$ & $\begin{array}{c}\text { S.aureus (+) } \\
\text { MIC }_{50}\end{array}$ & $\begin{array}{c}\text { S.typhi (-) } \\
\text { MIC }_{50}\end{array}$ & $\begin{array}{c}\text { P.aureginosa (-) } \\
\text { MIC }_{50}\end{array}$ & $\begin{array}{c}\text { E.coli (-) } \\
\text { MIC }_{50}\end{array}$ & $\begin{array}{c}\text { S. sonnei (-) } \\
\text { MIC }_{50}\end{array}$ \\
\hline 2a & $8.41 \pm 0.18$ & $16.78 \pm 0.22$ & $13.04 \pm 0.33$ & $12.99 \pm 0.24$ & $16.85 \pm 0.00$ & $7.65 \pm 0.11$ \\
2b & $18.17 \pm 0.17$ & $15.42 \pm 0.15$ & $11.05 \pm 0.32$ & $11.73 \pm 0.28$ & - & $7.39 \pm 0.77$ \\
$\mathbf{2 c}$ & $19.04 \pm 0.51$ & - & $12.27 \pm 0.35$ & $13.07 \pm 0.12$ & - & $16.03 \pm 0.11$ \\
$\mathbf{2 d}$ & $13.56 \pm 0.50$ & $18.62 \pm 0.29$ & $12.88 \pm 0.13$ & $14.31 \pm 0.22$ & $16.03 \pm 0.46$ & $13.91 \pm 0.17$ \\
$\mathbf{2 e}$ & - & $19.21 \pm 0.28$ & $14.38 \pm 0.00$ & $12.02 \pm 0.29$ & - & $10.91 \pm 0.32$ \\
$\mathbf{2 f}$ & $14.69 \pm 0.33$ & $13.58 \pm 0.21$ & $15.33 \pm 0.23$ & $14.55 \pm 0.01$ & - & $13.72 \pm 0.41$ \\
$\mathbf{2 g}$ & - & $7.65 \pm 0.21$ & $13.26 \pm 0.41$ & $12.01 \pm 0.21$ & $18.17 \pm 0.50$ & $10.98 \pm 0.23$ \\
$\mathbf{2 h}$ & - & - & - & - & - \\
Ampicilin & $11.66 \pm 0.14$ & $10.69 \pm 0.06$ & $10.85 \pm 0.16$ & $12.33 \pm 0.15$ & $11.32 \pm 0.13$ & $11.98 \pm 0.13$ \\
Gentamycin & $10.36 \pm 0.13$ & $8.42 \pm 0.12$ & $11.21 \pm 0.31$ & $10.89 \pm 0.11$ & $8.21 \pm 0.02$ & $9.31 \pm 0.18$ \\
Ciprofloxacin & $8.36 \pm 0.12$ & $9.42 \pm 0.11$ & $7.59 \pm 0.11$ & $11.03 \pm 0.10$ & $8.21 \pm 0.02$ & $7.31 \pm 0.08$ \\
\hline
\end{tabular}

The $\mathrm{H}-5$ of the pyridine ring appeared as a singlet in the range of 8.15 to $8.22 \mathrm{ppm}$. The carbonyl carbon in all ${ }^{13} \mathrm{C}$ NMR spectra gave a discrete signal in the range of 166.34 to $167.45 \mathrm{ppm}$. CHN analysis and HRMS also confirmed the structure of the prepared compounds.

\section{Antibacterial studies}

Procedure: The antibacterial activity was performed in sterile 96-wells microplates under aseptic environments ${ }^{14,15}$. The test samples with suitable solvents and dilutions were pipetted into wells $(20 \mu \mathrm{g} /$ well $)$. Overnight maintained fresh bacterial culture after suitable dilution with fresh nutrient broth was poured into wells $(180 \mu \mathrm{L})$. The initial absorbance of the culture was maintained between $0.12-0.19$ at $540 \mathrm{~nm}$. The total volume in each well was kept to $200 \mu \mathrm{L}$. The incubation was done at $37{ }^{\circ} \mathrm{C}$ for $16-24 \mathrm{~h}$ with lid on the microplate. The absorbance was measured at $540 \mathrm{~nm}$ using microplate reader, before and after incubation and the difference was noted as an index of bacterial growth. Ciprofloxacin, gentamycin and ampicilin were taken as standard. Minimum inhibitory concentration (MIC) was measured with suitable dilutions (5-30 $\mu \mathrm{g} /$ well) and results were calculated using EZ-Fit5 Perrella Scientific Inc. Amherst USA software and data expressed as $\mathrm{MIC}_{50}(\mu \mathrm{g} / \mathrm{mL})$. Results are mean of triplicate $(\mathrm{n}=3, \pm$ sem $)$.

Table- 2 shows the results of $\mathrm{MIC}_{50}$ studies of 6-aryl-3methyl-1-phenyl- $1 H$-pyrazolo[3,4- $b]$ pyridine-4-carbxylic acids (2a-2h) against six strains. Compound $\mathbf{2 a}$ showed comparable activity against the $B$. subtilis (+) and $S$. sonnei (-) to the standard ciprofloxacin. Compound $\mathbf{2 b}$ was found to show lower $\mathrm{MIC}_{50}$ value $\left(\mathrm{MIC}_{50}=11.05 \pm 0.32\right)$ against $S$. typhi (-) than the gentamycin $\left(\mathrm{MIC}_{50}=10.85 \pm 0.16\right)$. It is also found to be more active against $P$. aureginosa $(-)\left(\mathrm{MIC}_{50}=11.73 \pm\right.$ $0.28)$ than ampicilin $\left(\mathrm{MIC}_{50}=12.33 \pm 0.15\right)$. However it has a comparable $\mathrm{MIC}_{50 \mathrm{~b}}$ value $\left(\mathrm{MIC}_{50}=7.39 \pm 0.77\right)$ to the ciprofloxacin $\left(\mathrm{MIC}_{50}=7.31 \pm 0.08\right)$ against $S$. sonnei (-). Compound $2 \mathbf{e}$ is a little more active $\left(\mathrm{MIC}_{50}=12.02 \pm 0.29\right)$ than ampicilin $\left(\mathrm{MIC}_{50}=12.33 \pm 0.15\right)$ against $P$. aureginosa $(-)$. Compound $\mathbf{2 g}$ showed some significant results. It is found to be more active against $S$. aureus $(+)\left(\mathrm{MIC}_{50}=7.65 \pm 0.21\right)$ than gentamycin $\left(\mathrm{MIC}_{50}=8.42 \pm 0.12\right)$. It also showed lower $\mathrm{MIC}_{50}$ value against $P$. aureginosa $(-)\left(\mathrm{MIC}_{50}=12.01 \pm 0.21\right)$ than ampicilin $\left(\mathrm{MIC}_{50}=12.33 \pm 0.15\right)$. Moreover it is found to be more active against $S$. sonnei $(-)\left(\mathrm{MIC}_{50}=10.98 \pm 0.23\right)$ than ampicilin $\left(\mathrm{MIC}_{50}=11.98 \pm 0.13\right)$. So we can assume that this series of compound showed significant antibacterial activity.

\section{ACKNOWLEDGEMENTS}

The authors (TM, AN and MN) are indebted to the Higher Education Commission, Government of Pakistan for Indigenous Ph.D. Scholarships and IRSIP fellowships. One of the authors, MAK are thankful to the HEC for the Instrumental Analysis Support programme.

\section{REFERENCES}

1. R.R. Crenshaw, G.M. Luke and P. Siminoff, J. Med. Chem., 19, 262 (1976).

2. R.R. Crenshaw, G.M. Luke and P. Smirnoff, Canadian Patent, 10,32538 (1978).

3. M. Cheung, P.A. Harris, J.G. Badiang, G.E. Peckham, S.D. Chamberlain, M.J. Alberti, D.K. Jung, S.S. Harris, N.H. Bramson, A.H. Epperly, S.A. Stimpson and M.R. Peel, Bioorg. Med. Chem. Lett., 18, 5428 (2008).

4. S.A. Saggar, J.T. Sisko, T.J. Tucker, R.M. Tynebor, D.S. Su and N.J. Anthony, US Patent, 021,442 (2007).

5. M.R. Bell and J.H. Ackerman, US Patent, 4,920,128 (1990).

6. A. Straub, J.-P. Stasch, C. Alonso-Alija, J. Benet-Buchholz, B. Ducke, A. Feurer and C. Fürstner, Bioorg. Med. Chem. Lett., 11, 781 (2001).

7. M.N. Elnagdi, M.R.H. Elmoghayar and G.E.H. Elgemeie, Adv. Heterocycl. Chem., 41, 319 (1987).

8. R.G. Stein, J.H. Biel and T. Singh, J. Med. Chem., 13, 153 (1970).

9. R.G. Stein, J.H. Biel and T. Singh, J. Med. Chem., 13, 153 (1970).

10. A.M. Farghlay, N.S. Habib, M.A. Khalil and O.A. El-Sayed, J. Alexandria Pharm. Sci., 3, 90 (1989).

11. V.A. Chebanov, Y.I. Sakhno, S.M. Desenko, V.N. Chernenko, V.I. Musatov, S.V. Shishkina, O.V. Shishkin and C.O. Kappe, Tetrahedron, 63, 1229 (2007).

12. L.A.S. Mazzacaro, L.R.S. Dias, M.A. Khan, A.C.C. Freitas and A.W. Bhatti, $21^{\text {st }}$ Annual Meeting, Sociedade Brasileira da Quimica, Pocos de Caldas, MG, Brazil, Q0-143, May (1998).

13. L.G. Tensmeyer and C. Ainsworth, J. Org. Chem., 31, 1878 (1966).

14. M. Kaspady, V. Narayanaswamy, M. Raju and G. Rao, Drug Des. Discov., 6, 21 (2009).

15. C.-R. Yang, Y. Zhang, M.R. Jacob, S.I. Khan, Y.-J. Zhang and X.-C. Li, Antimicrob. Agents Chemother., 50, 1710 (2006). 